\title{
Massive stars exploding in a He-rich circumstellar medium - VIII. PSN J07285387+3349106, a highly reddened supernova Ibn
}

\author{
A. Pastorello ${ }^{1 \star}$, L. Tartaglia ${ }^{1,2}$, N. Elias-Rosa ${ }^{1}$, A. Morales-Garoffolo ${ }^{3}$, \\ G. Terreran ${ }^{1,4}$, S. Taubenberger ${ }^{5,6}$, U. M. Noebauer ${ }^{6}$, S. Benetti ${ }^{1}$, E. Cappellaro ${ }^{1}$, \\ F. Ciabattari ${ }^{7}$, M. Dennefeld ${ }^{8}$, A. Dimai ${ }^{9}$, E. E. O. Ishida ${ }^{6}$, A. Harutyunyan ${ }^{10}$, \\ S. Leonini ${ }^{11}$, P. Ochner ${ }^{1}$, J. Sollerman ${ }^{12}$, F. Taddia ${ }^{12}$, S. Zaggia ${ }^{1}$ \\ ${ }^{1}$ INAF - Osservatorio Astronomico di Padova, Vicolo dell'Osservatorio 5, I-35122 Padova, Italy \\ ${ }^{2}$ Dipartimento di Fisica e Astronomia, Università degli Studi di Padova, Vicolo dell'Osservatorio 3, I-35122 Padova, Italy \\ ${ }^{3}$ Institut de Ciències de l'Espai (CSIC-IEEC), Campus UAB, Camí de Can Magrans S/N, 08193 Cerdanyola (Barcelona), Spain \\ ${ }^{4}$ Astrophysics Research Centre, School of Mathematics and Physics, Queen's University Belfast, Belfast BT7 1NN, UK \\ ${ }^{5}$ European Organisation for Astronomical Research in the Southern Hemisphere (ESO), Karl-Schwarzschild-Str. 2, D-85748 \\ Garching bei München, Germany \\ ${ }^{6}$ Max-Planck-Institut für Astrophysik, Karl-Schwarzschild-Str. 1, D-85748 Garching bei München, Germany \\ ${ }^{7}$ Osservatorio Astronomico di Monte Agliale, Via Cune Motrone, Borgo a Mozzano, Lucca, I-55023, Italy \\ ${ }^{8}$ Institut d'Astrophysique de Paris, CNRS, and Universite Pierre et Marie Curie, 98 bis Boulevard Arago, 75014, Paris, France \\ ${ }^{9}$ Osservatorio Astronomico del Col Druscié, I-32043 Cortina d'Ampezzo, Italy \\ ${ }^{10}$ Fundación Galileo Galilei-INAF, Telescopio Nazionale Galileo, Rambla Jos Ana Fernández Pérez 7, E-38712 Breña Baja, TF, Spain \\ ${ }^{11}$ Osservatorio Astronomico Provinciale di Montarrenti, S.S. 73 Ponente, I-53018, Sovicille, Siena, Italy \\ ${ }^{12}$ The Oskar Klein Centre, Department of Astronomy, Stockholm University, AlbaNova, 10691 Stockholm, Sweden
}

Accepted YYYY Month DD. Received YYYY Month DD; in original form YYYY Month DD

\begin{abstract}
We present spectroscopic and photometric observations for the Type Ibn supernova (SN) dubbed PSN J07285387+3349106. Using data provided by amateur astronomers, we monitored the photometric rise of the SN to maximum light, occurred on 2015 February 18.8 UT $\left(\mathrm{JD}_{\text {max }}(V)=2457072.0 \pm 0.8\right)$. PSN J07285387+3349106 exploded in the inner region of an infrared luminous galaxy, and is the most reddened SN Ibn discovered so far. We apply multiple methods to derive the total reddening to the $\mathrm{SN}$, and determine a total colour excess $\mathrm{E}(B-V)_{t o t}=0.99 \pm 0.48 \mathrm{mag}$. Accounting for the reddening correction, which is affected by a large uncertainty, we estimate a peak absolute magnitude of $M_{V}=-20.30 \pm$ 1.50. The spectra are dominated by continuum emission at early phases, and $\mathrm{He} \mathrm{I}$ lines with narrow P-Cygni profiles are detected. We also identify weak Fe III and N II features. All these lines show an absorption component which is blue-shifted by about $900-1000 \mathrm{~km} \mathrm{~s}^{-1}$. The spectra also show relatively broad He I line wings with low contrast, which extend to above $3000 \mathrm{~km} \mathrm{~s}^{-1}$. From about 2 weeks past maximum, broad lines of O I, Mg II and the $\mathrm{Ca}$ II near-infrared triplet are identified. The composition and the expansion velocity of the circumstellar material, and the presence of $\mathrm{He}$ I and $\alpha$-elements in the SN ejecta indicate that PSN J07285387+3349106 was produced by the core-collapse of a stripped-envelope star. We suggest that the precursor was WNE-type Wolf-Rayet star in its dense, He-rich circumstellar cocoon.
\end{abstract}

Key words: supernovae: general, supernovae: individual: PSN J07285387+3349106, SN 2006jc, SN 2010al, ASASSN-15ed, stars: Wolf-Rayet

\section{INTRODUCTION}

Type Ibn supernovae (SNe Ibn) are a class of transients whose spectra show narrow lines of $\mathrm{He} \mathrm{I}$ in emission and weak (or no) evidence of $\mathrm{H}$ lines (Matheson et al. 2000; Pastorello et al. 2008a). This is interpreted as evidence for the presence of a He-rich circumstel- 
lar medium (CSM). In contrast to SNe IIn, whose CSM is dominated by $\mathrm{H}$, in $\mathrm{SNe}$ Ibn the outer environment is almost $\mathrm{H}$-free. This implies that the progenitor stars of SNe Ibn already lost their $\mathrm{H}$ envelope, and also (part of) the He-rich layers at the time of their terminal explosion.

As recent publications in the literature have pointed out, the number of SN Ibn discoveries has significantly grown in the past few years. Modern surveys with very large fields of view have led to an increase in detection rates and have removed the galaxy selection biases. With deeper imaging, and hence an increased sampled volume, $\mathrm{SNe}$ Ibn have also been discovered at relatively high redshifts. Finally, more accurate typing criteria have significantly decreased the risk of mis-classifications. In fact, the discoveries of transitional SNe Ibn (including the so-called SNe Ibn/IIn, such as SNe 2005la and 2011hw, that both show signatures of some circumstellar $\mathrm{H}$ along with He, Pastorello et al. 2008b, 2015a; Smith et al. 2012; Modjaz et al. 2014; Bianco et al. 2014) or SNe Ibn that evolve into SNe Ib (e.g. ASASSN-15ed and SN 2015G, Pastorello et al. 2015b; Fraser et al. 2015), have shown that the classification of an object as a Type Ibn $\mathrm{SN}$ is sensitive to the epoch at which the spectrum is obtained. As a consequence, a proper classification requires goodquality spectra obtained at multiple epochs.

A major problem in SN Ibn identification is that they usually explode in star-forming environments (Taddia et al. 2015; Pastorello et al. 2015c), hence their spectra are heavily contaminated by the complex host galaxy background. The rapid post-peak luminosity decline furthermore complicates their accurate characterization. In fact, most objects faded below the detection limit of optical telescopes within about 2 months (Matheson et al. 2000; Pastorello et al. 2008b, 2015d; Gorbikov et al. 2014), with at least one observed exception (OGLE-2012-SN-006, which experienced an unusually slow photometric evolution, Pastorello et al. 2015d).

The reason for the rapid evolution in the optical bands of most $\mathrm{SNe}$ Ibn is debated. In several cases, the rapid decline of the optical light curves was linked to a prompt condensation of dust in SN ejecta or in a cool dense shell (CDS, e.g. Chugai et al. 2004; Kankare et al. 2012; Stritzinger et al 2012). In SN 2006jc, the prototype of this family, this process was directly observed (Smith et al. 2008; Mattila et al. 2008; Di Carlo et al. 2008; Nozawa et al. 2008; Sakon et al. 2009). SN 2006jc is a milestone for a number of reasons. First of all, it is still the only SN Ibn for which a pre-SN outburst was observed (by the amateur astronomer K. Itagaki) two years before the $\mathrm{SN}$ explosion (Nakano et al. 2006; Pastorello et al. 2007). In addition, SN 2006jc was well monitored in the ultra-violet (UV), optical and near-infrared (NIR) bands since it was hosted by a nearby galaxy (Foley et al. 2007; Pastorello et al. 2007, 2008a; Di Carlo et al. 2008; Anupama et al. 2009; Modjaz et al. 2014; Bianco et al. 2014). For this reason, SN 2006jc is still the object that provided the most detailed information on this $\mathrm{SN}$ family (Chugai 2009, and references therein).

A factor that is expected to affect the detection rate of SNe Ibn, which typically explode in star-forming galaxies, is their possible location in a dusty regions. However, no SN Ibn affected by significant reddening has been discovered so far. The first opportunity to monitor an object of this class with strong line-of-sight extinction is PSN J07285387+3349106. The discovery of this transient by the Lick Observatory Supernova Search (LOSS) 1 and its early followup information have been reported on the CBAT "Transient Object

1 http://w.astro.berkeley.edu/bait/public_html/kait.html

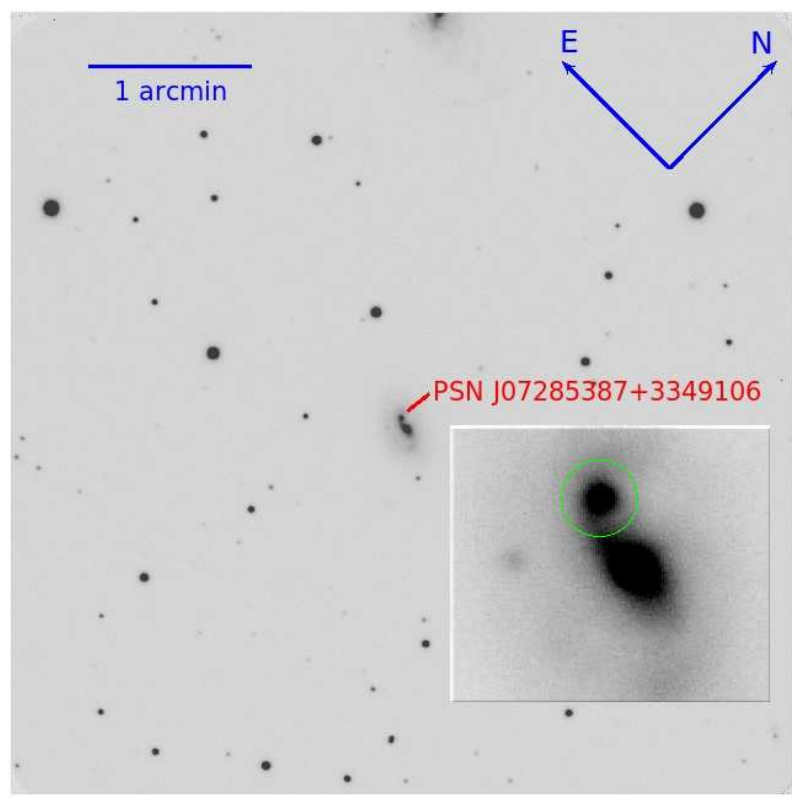

Figure 1. PSN J07285387+3349106 and its host galaxy. $R$-band image taken on 2015 October 2 with ALFOSC, mounted at the Nordic Optical Telescope. The insert shows a blow-up of the SN region.

Followup Reports" pages. PSN J07285387+3349106 was classified by Ochner et al. (2015) as a young Type Ibn event based on the presence of narrow emission lines of He I. In addition, the detection of the strong, unresolved $\mathrm{Na}$ I doublet $\lambda \lambda 5889,5895$ (Na ID) in absorption at the redshift of the host galaxy $(\mathrm{z}=0.01379)$ led Ochner et al. to conclude that the SN was significantly reddened, with $\mathrm{E}(B-V)_{\text {tot }} \sim 1$ mag. Multi-band optical photometry of PSN J07285387+3349106 has been published by Tsvetkov et al. (2015).

This article is the eighth of a series whose main goals are to illustrate the variety of physical properties that characterize the Type Ibn SNe family, and to constrain the nature of their progenitor stars. This paper is arranged as follows: In Section 2 we describe the main properties of the galaxy hosting PSN J07285387+3349106. In Section 3 we compute the total reddening in the $\mathrm{SN}$ direction. In Section 4 we present the observations and illustrate the data reduction procedures. Sections 5 and 6 analyse the SN light curve and the spectroscopic evolution, respectively. A discussion and a summary follow in Section 7

\section{THE HOST GALAXY}

The galaxy hosting PSN J07285387+3349106, NGC 2388 (Figure 11), is classified as an Sb-type in Hyperled 3 (Makarov et al. 2014), and is apparently one member of the galaxy group WBL 142 , which is composed of three galaxies within a radius of 82 arcmin (Zwicky et al. 1961-1968), whose mean redshift is $\mathrm{z} \sim 0.0135$ (White et al. 1999). NGC 2388 has been classified as a possible luminous infrared galaxy (LIRG) by Sanders et al. (2003). As it has

2 http://www.cbat.eps.harvard.edu/unconf/followups/J07285387+3349106.html

3 http://leda.univ-lyon1.fr/ 
been detected from the UV to the radio wavelengths, its spectral energy distribution is well constrained (Brown et al. 2014). In addition, our SN follow-up spectra (see Section 6) show very prominent emission lines attributed to the host galaxy. All of this suggests that NGC 2388 is a star-forming galaxy.

The radial velocity of NGC 2388 corrected for Local Group infall onto Virgo is $\mathrm{v}_{\text {Vir }}=4255 \mathrm{~km} \mathrm{~s}^{-1}$ (from Hyperleda, Makarov et al. 2014). Adopting a standard cosmology with $\mathrm{H}_{0}=$ $73 \mathrm{~km} \mathrm{~s}^{-1} \mathrm{Mpc}^{-1}, \Omega_{M}=0.27, \Omega_{\Lambda}=0.73$, we obtain a luminosity distance of $58.9 \pm 4.1 \mathrm{Mpc}$ (see Wright 2006, for information on the cosmology calculator), hence a distance modulus $\mu=33.85 \pm$ $0.15 \mathrm{mag} 4$

As the total apparent magnitude of NGC 2388 provided by Hyperleda is $B=14.66 \pm 0.32$, the total absolute $B$-band magnitude corrected for Galactic and internal extinction (following the prescriptions of Hyperleda) is -19.76 . Using the luminosity vs. metallicity relations provided by Tremonti et al. (2004), we estimate an integrated oxygen abundance of $12+\log (\mathrm{O} / \mathrm{H})=8.91$ (dex) for NGC 2388. The SN exploded in the proximity of a spiral arm of the host galaxy, about 6 arcsec East and 2 arcsec North of the nucleus. Accounting for the SN position, and following Pastorello et al. (2015c), we estimate the $R_{0, S N} / R_{25}$ ratid 5 to be 0.26 . This allows us to compute the oxygen abundance at the SN position, assuming a radial dependence as in Pilyugin et al. (2004). We obtain $12+\log (\mathrm{O} / \mathrm{H})_{S N}=8.81(\mathrm{dex})$, which is slightly above the average oxygen abundance estimated at the SN location for the host galaxies of SNe Ibn by Pastorello et al. 2015c, $8.63 \pm 0.42$ dex) and Taddia et al. (2015, $8.45 \pm 0.10 \mathrm{dex}$ ).

The analysis of the host galaxy of PSN J07285387+3349106 confirms the results of the above systematic studies, namely that there is no evidence of SNe Ibn preferentially exploding in metalpoor environments.

\section{THE LINE-OF-SIGHT EXTINCTION TO PSN J07285387+3349106}

As stated by Ochner et al. (2015), PSN J07285387+3349106 is significantly extinguished, and hence the estimate of its reddening is of utmost importance. The Milky Way contribution to the total reddening is modest. Following the revised Galactic reddening estimates of Schlafly \& Finkbeiner (2011) and assuming a Cardelli et al (1989) reddening law with $\mathrm{R}_{V}=3.1$, we obtain $\mathrm{E}(B-V)_{M W}=0.05 \mathrm{mag}$.

Obtaining the $\mathrm{SN}$ extinction in the host galaxy is more complicated. For this purpose, we measure the equivalent width (EW) of the narrow interstellar Na I $\lambda \lambda 5890,5896$ (Na ID) in the SN spectra to improve the preliminary estimate reported in Ochner et al. (2015). Unfortunately, this measurement is uncertain, because the region is affected by the the presence of a SN CSM emission line (He I $\lambda 5876$, see Section 6 which increases in strength with time. Since the He I line is still very weak in our earliest spectrum, it can be used to derive a more robust estimate despite its relatively poor signal-to-noise $(\mathrm{S} / \mathrm{N})$. From this, we measure an $\mathrm{EW}=6.5 \pm$ $0.5 \AA$ for the interstellar Na ID. According to the empirical relation

\footnotetext{
4 The errors on the distance and distance modulus were computed accounting for the uncertainties on the recessional velocity, the Local Group infall correction, and the cosmological parameters, following the prescriptions of the NED database, https://ned.ipac.caltech.edu/.

${ }^{5} \mathrm{R}_{S N}$ is the deprojected position of the $\mathrm{SN}$, while $\mathrm{R}_{25}$ is the isophotal radius for the $B$-band surface brightness of $25 \mathrm{mag} \operatorname{arcsec}^{2}$.
}

provided by Turatto et al. (2003), we obtain $\mathrm{E}(B-V)_{\text {host }}=1.03$ mag. However, it is known that the relation between Na ID EW and $\mathrm{E}(B-V)$ has a large dispersion, especially when the interstellar extinction is high (see, e.g., Poznanski et al. 2011). In order to account for this, we note that for EW larger than $4 \AA$, the SN sample of Poznanski et al. spans a range of $A_{V}$ values from about 0.5 to 3 mag. Therefore $\Delta A_{V} \approx 1.25$ max can be considered an indicative guess for the error on the reddening with this method. Hence, $\mathrm{E}(B-V)_{\text {tot }}=1.08 \pm 0.40 \mathrm{mag}$ is our estimate for the total interstellar extinction to PSN J07285387+3349106 using the interstellar Na ID.

As the above method is affected by a large uncertainty, we estimate the amount of extinction using alternative methods. First, we follow Calzetti et al. (1994) and compute the total reddening to the SN through the host galaxy Balmer lines decrement. The decrement is measured using different spectra extracted in regions very close to the $\mathrm{SN}$ position. With this approach, we infer values of $\mathrm{E}(B-V)_{t o t} \approx 1$ to $1.5 \mathrm{mag}$ (with a weighted average of 1.34 $\pm 0.31 \mathrm{mag})$.

Another method to compute the foreground reddening, which is widely used for Type Ia SNe (e.g., Phillips et al. 1999), is through the comparison of SN colour curves at defined epochs. In the case of Type Ibn SNe, this method is limited by the large heterogeneity of this class of transients. However, using the intrinsic colours of a limited number of SNe Ibn which are photometrically similar to PSN J07285387+3349106 (see Section 5 for more details), we obtain a somewhat smaller value for the reddening, viz. $\mathrm{E}(B-V)_{t o t}$ $=0.81 \pm 0.21 \mathrm{mag}$.

A weighted average of these three methods provides as best estimate of the total reddening $\mathrm{E}(B-V)_{t o t}=0.99 \pm 0.48 \mathrm{mag}$, which will be used throughout the paper.

\section{OBSERVATIONS AND DATA REDUCTION}

Our spectroscopic and photometric follow-up campaigns were triggered soon after the classification. Early-time photometry data and pre-discovery observations of the field were gathered a posteriori thanks to the routine unfiltered observations of NGC 2388 by a number of amateur astronomers in the framework of the Italian Supernovae Search Program (ISSP 6 . Details on the observations obtained using 30 - to $50-\mathrm{cm}$ telescopes available to the ISSP collaboration are provided in the footnotes of Table 1

Standard photometric follow-up of PSN J07285387+3349106 was performed using the following instruments: the 1.82-m Copernico Telescope of the INAF - Padova Observatory at Mt. Ekar (Asiago, Italy) equipped with AFOSC; the 2.56-m Nordic Optical Telescope (NOT) with ALFOSC and the 3.58-m Telescopio Nazionale Galileo (TNG) equipped with DOLORES (LRS), both located at Roque de los Muchachos Observatory (La Palma, Canary Islands, Spain); the 0.8-m Joan Oró Telescope (TJO) of the Observatori Astronòmic del Monsec (Catalonia, Spain) equipped with MEIA; the INAF 0.6-m Rapid Eye Mount (REM) Ritchey-Chrétien reflector Telescope hosted by the European Southern Observatory (ESO, La Silla, Chile) equipped with ROSS2.

Photometric images were pre-reduced using standard tasks

\footnotetext{
6 http://italiansupernovae.org the ISSP is a coordinated SN search involving several Italian Amateur Observatories located in Veneto, Toscana and Lombardia. Since 2011 it has led to the discovery of about $80 \mathrm{SNe}$.
} 
Table 1. Photometric data and associated errors for PSN J07285387+3349106.

\begin{tabular}{|c|c|c|c|c|c|c|c|c|c|}
\hline Date & JD & $B$ & $V$ & $u$ & $g$ & $r$ & $i$ & $z$ & Instrument \\
\hline 20141212 & 2457003.53 & - & $>19.15$ & - & - & $>19.03$ & - & - & 1 \\
\hline 20141223 & 2457014.42 & - & $>19.01$ & - & - & $>18.80$ & - & - & 2 \\
\hline 20141226 & 2457018.46 & - & $>19.88$ & - & - & $>19.76$ & - & - & 1 \\
\hline 20141227 & 2457018.63 & - & $>20.41$ & - & - & $>20.19$ & - & - & 3 \\
\hline 20150107 & 2457030.49 & - & $>19.10$ & - & - & $>18.90$ & - & - & 2 \\
\hline 20150112 & 2457035.49 & - & $>20.19$ & - & - & $>19.97$ & - & - & 3 \\
\hline 20150209 & 2457063.47 & - & $18.84(0.35)$ & - & - & $18.62(0.26)$ & - & - & 3 \\
\hline 20150210 & 2457064.45 & - & $18.29(0.38)$ & - & - & $18.14(0.30)$ & - & - & 4 \\
\hline 20150214 & 2457068.37 & - & $17.09(0.29)$ & - & - & $16.73(0.32)$ & - & - & 5 \\
\hline 20150217 & 2457071.33 & - & - & - & - & $16.41(0.24)$ & - & - & AFOSC \\
\hline 20150217 & 2457071.34 & - & $16.65(0.24)$ & - & - & $16.46(0.26)$ & - & - & 1 \\
\hline 20150218 & 2457072.26 & $17.46(0.09)$ & $16.62(0.09)$ & - & - & - & - & - & AFOSC \\
\hline 20150219 & 2457072.39 & - & - & $18.69(0.05)$ & $17.28(0.03)$ & $16.48(0.03)$ & $16.20(0.04)$ & $16.01(0.06)$ & AFOSC \\
\hline 20150220 & 2457073.52 & $17.77(0.14)$ & $16.71(0.09)$ & $18.85(0.08)$ & $17.30(0.06)$ & $16.53(0.06)$ & $16.24(0.04)$ & - & AFOSC \\
\hline 20150222 & 2457075.58 & - & - & - & $17.45(0.09)$ & $16.58(0.09)$ & $16.39(0.08)$ & $16.00(0.16)$ & ROSS2 \\
\hline 20150222 & 2457076.46 & $17.99(0.03)$ & $16.92(0.02)$ & - & - & $16.71(0.03)$ & $16.47(0.05)$ & - & ALFOSC \\
\hline 20150223 & 2457077.48 & $18.09(0.07)$ & $17.11(0.06)$ & $19.46(0.08)$ & $17.69(0.06)$ & $16.79(0.08)$ & $16.49(0.06)$ & - & LRS \\
\hline 20150225 & 2457078.54 & - & - & - & $17.83(0.10)$ & $16.85(0.14)$ & $16.58(0.09)$ & $16.17(0.13)$ & ROSS2 \\
\hline 20150225 & 2457078.58 & $18.20(0.07)$ & $17.17(0.09)$ & - & - & - & - & - & LRS \\
\hline 20150227 & 2457081.36 & $18.70(0.20)$ & $17.64(0.21)$ & - & - & - & - & - & MEIA \\
\hline 20150228 & 2457082.39 & - & - & - & - & $17.30(0.25)$ & - & - & LRS \\
\hline 20150301 & 2457082.54 & - & - & - & $18.09(0.26)$ & $17.30(0.15)$ & $16.99(0.15)$ & $16.39(0.21)$ & ROSS2 \\
\hline 20150302 & 2457083.59 & - & - & - & $18.25(0.23)$ & $17.54(0.20)$ & $17.11(0.16)$ & $16.47(0.26)$ & ROSS2 \\
\hline 20150302 & 2457084.37 & $19.12(0.38)$ & $18.13(0.23)$ & - & - & - & - & - & MEIA \\
\hline 20150303 & 2457085.47 & - & - & - & - & $17.80(0.22)$ & - & - & ALFOSC \\
\hline 20150304 & 2457085.67 & - & - & - & $>16.99$ & $>16.42$ & $17.43(0.53)$ & $>15.55$ & ROSS2 \\
\hline 20150304 & 2457086.32 & $>17.99$ & - & - & - & - & - & - & MEIA \\
\hline 20150305 & 2457086.53 & - & - & - & $18.96(0.36)$ & $18.02(0.23)$ & $17.59(0.19)$ & $17.13(0.29)$ & ROSS2 \\
\hline 20150307 & 2457089.35 & - & $19.21(0.42)$ & - & - & $18.96(0.39)$ & - & - & 3 \\
\hline 20150308 & 2457090.35 & - & $19.59(0.42)$ & - & - & $19.27(0.35)$ & - & - & 3 \\
\hline 20150310 & 2457091.54 & - & - & - & $20.35(0.51)$ & $20.00(0.59)$ & $19.34(0.46)$ & $18.97(0.38)$ & ROSS2 \\
\hline 20150310 & 2457092.30 & - & $>19.04$ & - & - & $>18.83$ & - & - & 2 \\
\hline 20150310 & 2457092.47 & $21.89(0.23)$ & $20.42(0.22)$ & - & - & $20.13(0.30)$ & $19.69(0.34)$ & - & ALFOSC \\
\hline 20150311 & 2457093.30 & - & - & $22.51(0.54)$ & $21.08(0.20)$ & $20.49(0.19)$ & $19.89(0.14)$ & $19.39(0.12)$ & AFOSC \\
\hline 20150312 & 2457094.33 & - & $>20.23$ & - & $21.35(0.19)$ & $20.60(0.18)$ & $20.04(0.14)$ & $19.58(0.15)$ & AFOSC \\
\hline 20150330 & 2457112.34 & - & $>20.02$ & - & - & $>19.78$ & - & - & 3 \\
\hline 20150330 & 2457112.34 & - & $>18.01$ & - & - & $>17.85$ & - & - & 1 \\
\hline
\end{tabular}

1- 0.50-m Newton telescope (Lotti) equipped with a FLI Proline 4710 camera with E2V CCD47-10 (Osservatorio Astronomico di Monte Agliale, Borgo a Mozzano, Lucca, Italy); 2- 0.28-m Schmidt-Cassegrain telescope (Vittore Maioni) with a SBIG ST-8 3 CCD Camera (Osservatorio Astronomico del Col Druscié, Cortina, Belluno, Italy); 3- 0.53-m Ritchey-Chrétien telescope equipped with an Apogee Alta U47 camera with E2V CCD47-10 (Osservatorio Astronomico Provinciale di Montarrenti, Siena, Italy); 4- 0.40-m Newton Marcon telescope equipped with an ATIK 428EX camera with Sony ICX674 CCD (private observatory; obs. Paolo Campaner, Ponte di Piave, Treviso, Italy); 5- 0.14-m TEC refractor telescope equipped with an ATIK 460EX camera with Sony ICX694ALG CCD and Luminance filter (private observatory; obs. Manfred Mrotzek, Buxtehude, Germany).

Table 2. Log of spectroscopic observations of PSN J07285387+3349106.

\begin{tabular}{ccccccc}
\hline Date & JD & Phase $(\mathrm{d})^{\ddagger}$ & Instrumental configuration & Exposure time $(\mathrm{s})$ & Range $(\AA)$ & Resolution $(\AA)$ \\
\hline $2015 / 02 / 17$ & 2457071.35 & -0.6 & 1.82 -m Copernico + AFOSC + gm4 & 1800 & $3500-8190$ & 13 \\
$2015 / 02 / 18$ & 2457072.25 & +0.3 & 1.82 -m Copernico + AFOSC + gm4 + VPH6 & $1800+1800$ & $3550-9270$ & $14+15$ \\
$2015 / 02 / 19$ & 2457073.49 & +1.5 & $1.82-\mathrm{m}$ Copernico + AFOSC + gm4 & $2 \times 1800$ & $3440-8170$ & 13 \\
$2015 / 02 / 22$ & 2457076.48 & +4.5 & $2.56-\mathrm{m}$ NOT + ALFOSC + gm4 & 1800 & $3330-9100$ & 18 \\
$2015 / 02 / 23$ & 2457077.43 & +5.4 & $3.58-\mathrm{m}$ TNG + LRS + LRB + LRR & $1500+1500$ & $3450-10190$ & $10+10$ \\
$2015 / 02 / 25$ & 2457078.60 & +6.6 & $3.58-\mathrm{m}$ TNG + LRS + LRB & 1029 & $3450-8060$ & 10 \\
$2015 / 02 / 28$ & 2457082.45 & +10.5 & $3.58-\mathrm{m}$ TNG + LRS + LRB & 1800 & $3450-8060$ & 10 \\
$2015 / 03 / 03$ & 2457085.49 & +13.5 & $2.56-\mathrm{m}$ NOT + ALFOSC + gm4 & 2700 & $3500-9110$ & 18 \\
$2015 / 03 / 11$ & 2457092.51 & +20.5 & $2.56-\mathrm{m}$ NOT + ALFOSC + gm4 $4300-9110$ & $2 \times 2700$ & 3300 \\
\hline
\end{tabular}

$\ddagger$ The phases are with respect to the $V$-band maximum light $\left(\mathrm{JD}_{\max }(V)=2457072.0\right)$. 


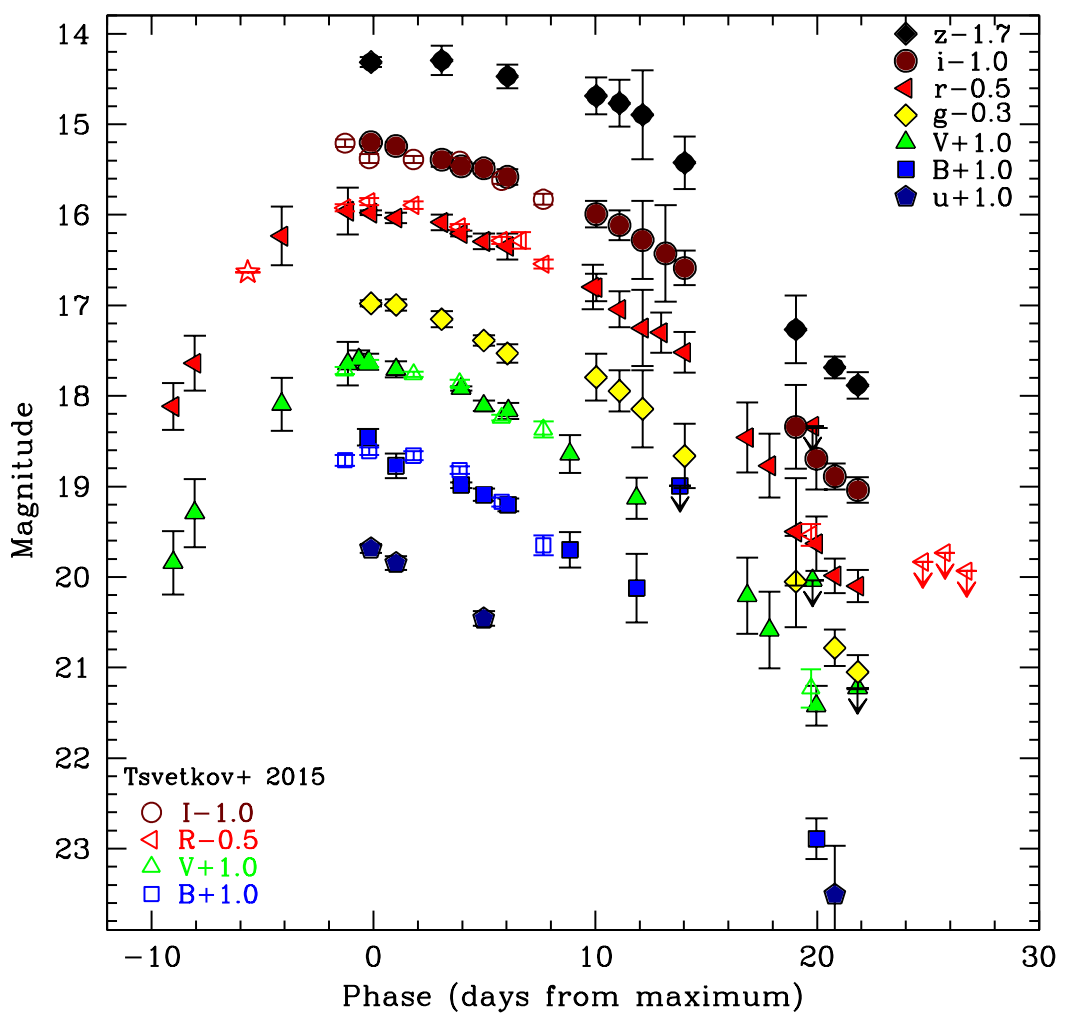

Figure 2. Multi-band light curves of PSN J07285387+3349106: our observations (filled symbols) are compared with those published by Tsvetkov et al. (2015, open symbols). A constant zeropoint correction has been applied to the Johnson-Cousins $R$ - and $I$-band data of Tsvetkov et al. (2015) to convert the magnitudes into the Sloan $r$ - and $i$ system. The corrections terms, $\Delta R=0.23 \pm 0.03$ mag and $\Delta I=0.47 \pm 0.03$ mag, have been obtained by computing the synthetic photometry from the available spectra of PSN J07285387+3349106. The starred symbol represents the scaled discovery photometry from LOSS.

available in IRAF7. The pre-reduction steps include overscan, bias and flat-field corrections, and final trimming of the useless image regions. Using the dedicated PYTHON pipeline SNOOPY (Cappellaro 2014) 8 we then astrometrically registered the images, extracted a number of stellar sources in the images and measured their instrumental magnitudes using a PSF-fitting technique. As the $\mathrm{SN}$ was located in a complex region inside the host galaxy, a properly removal of the background contribution using templates available in public archives (e.g. SDSS for the Sloan-band images) was required. The $\mathrm{SN}$ magnitude was measured after the host galaxy contamination was removed.

The magnitudes of a number of stars in the field of PSN J07285387+3349106 are available in the SDSS catalogue. These were used to estimate zeropoints and colour terms for all nights for which Sloan-band observations (in $u, g, r, i$, and $z$ ) were obtained. The Johnson $B$ and $V$ magnitudes were instead calibrated by converting the Sloan magnitudes of the stars in the SN field to Johnson magnitudes, following Chonis \& Gaskell (2008).

7 IRAF is distributed by the National Optical Astronomy Observatory, which is operated by the Association of Universities for Research in Astronomy (AURA) under cooperative agreement with the National Science Foundation.

${ }^{8}$ SNOOPY is a collection of PYRAF programs and other widely used public tools (e.g. DAOPHOT, SEXTRACTOR, HOTPANTS).
Early unfiltered data, kindly provided by amateur astronomers, were converted to Johnson $V$ and Sloan $r$ magnitudes. The calibrated SN magnitudes are listed in Table 1 The reported errors account for the uncertainties in the instrumental magnitudes and the photometric calibration. Information on the instruments used by amateur astronomers is provided in the footnotes of Table 1

Spectroscopic data, covering a 3-week temporal window starting at maximum light, were obtained with some of the instruments mentioned above, including AFOSC, mounted on the Copernico Telescope, LRS on the TNG and ALFOSC on the NOT. These data were processed using standard IRAF tools. Two-dimensional spectra were bias, flat-field and overscan corrected, afterwards onedimensional spectra were extracted from the images. The spectra were then wavelength calibrated using arc spectra obtained with the same instrumental configuration as the SN spectra. The wavelength calibration was accurately verified through a cross-check with the position of selected night sky lines. When necessary, we applied a constant wavelength shift. The flux calibration was performed using spectra of flux standard stars observed in the same night as the $\mathrm{SN}$, or using instrumental sensitivity functions for the same instrumental set-up available in our archive. Then, using the available broad-band photometry, the flux calibration of the SN spectra was verified and, in case of a discrepancy with the photometry, the spectra were multiplied by a constant factor. Contamination from $\mathrm{O}_{2}$ 

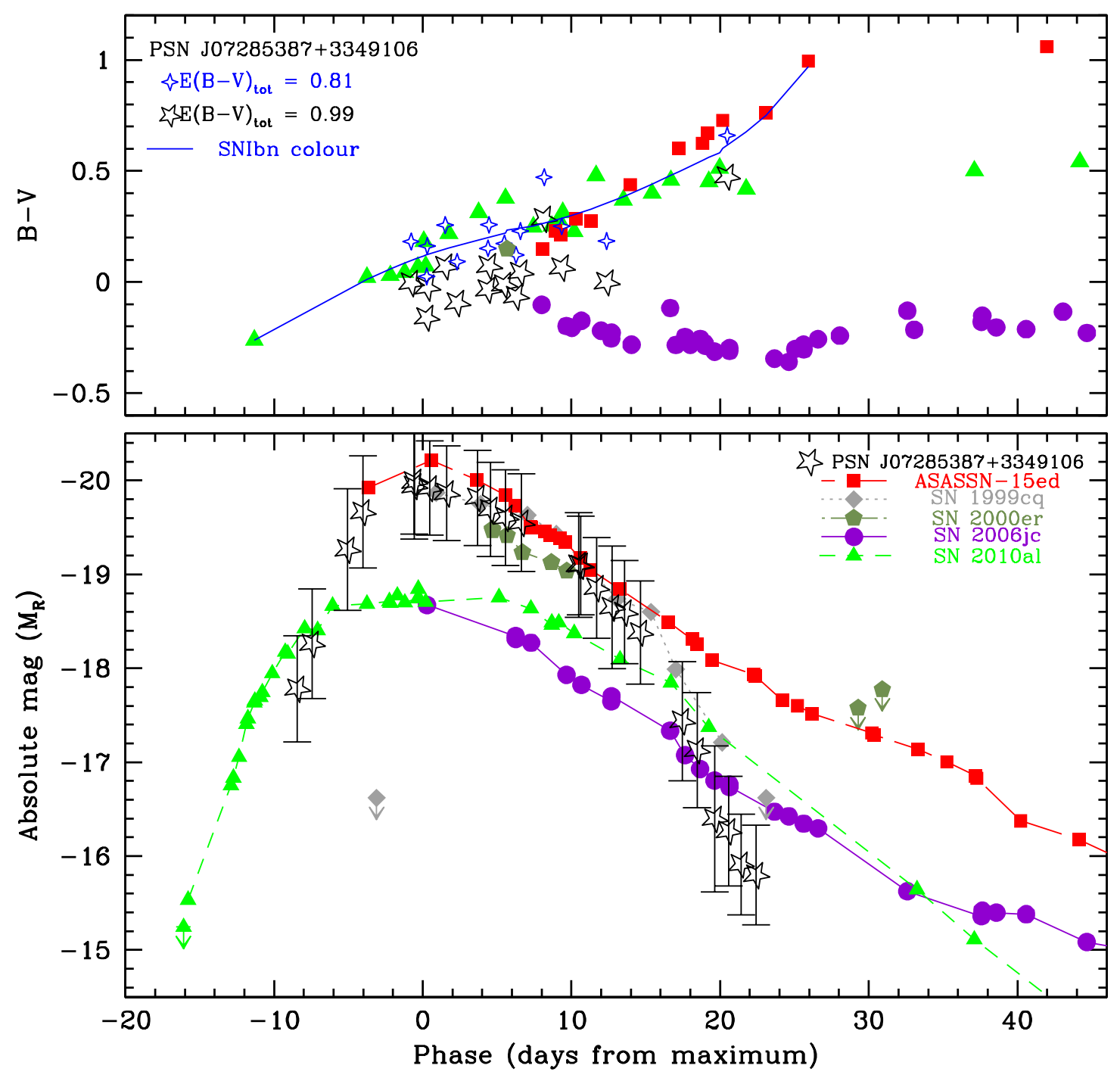

Figure 3. Top - Comparison of the $B-V$ colour evolution of PSN J07285387+3349106 with those of a sub-sample of SNe Ibn, including the photometrically similar SNe 2000er (Pastorello et al. 2008a) and ASASSN-15ed (Pastorello et al. 2015b), and the well-followed SNe 2006jc (Pastorello et al. 2007; Folev et al. 2007) and 2010al (Pastorello et al. 2015a). The blue solid line represents our SN Ibn colour template (see text). We show the colour evolution of PSN J07285387+3349106 for two values of $\mathrm{E}(B-V)_{\text {tot }}$ : $0.99 \mathrm{mag}$, as adopted throughout the paper, and 0.81 mag, obtained assuming that PSN J07285387+3349106 has the same colour evolution as the SN Ibn colour template. Bottom - Comparison of the $R$-band absolute light curve of PSN J07285387+3349106 (obtained from the Sloan-r light curve scaled by -0.23 mag and corrected for $\mathrm{E}(B-V)_{\text {tot }}=0.99$ mag), with those of the same sample as above plus SN 1999cq (Matheson et al. 2000). The LOSS discovery magnitude of PSN J07285387+3349106 is also included. The error bars account for the uncertainties of the photometric data, the interstellar extinction and the distance modulus.

and $\mathrm{OH}$ telluric bands was removed using the spectra of standard stars. Information on the spectra of PSN J07285387+3349106 and the instrumental configurations is listed in Table 2

\section{LIGHT CURVE}

In Figure 2 we compare our light curves of PSN J07285387+3349106 with those published by Tsvetkov et al. (2015). The Johnson-Cousins $R$ and $I$-band data of Tsvetkov et al. (2015) are scaled by a constant to match our Sloan $r$ and $i$-band photometry. These zeropoint corrections, $\Delta R=0.23 \pm 0.03$ mag and $\Delta I=0.47 \pm 0.03 \mathrm{mag}$, have been computed through synthetic photometry obtained from our spectra. The resulting match between our data and those of Tsvetkov et al. is fairly good.

Early-time unfiltered magnitudes from amateur astronomers, scaled to the Sloan $r$ and the Johnson $V$ systems, allow us to estimate the epochs and the apparent magnitudes at maximum in these 2 bands. Using a low-order polynomial fit to the light curves, we obtain: $V_{\max }=16.63 \pm 0.18\left(\right.$ on $\left.\mathrm{JD}_{\max }(V)=2457072.0 \pm 0.8\right)$ and $r_{\max }=16.45 \pm 0.15$ (on $\left.\mathrm{JD}_{\max }(r)=2457071.9 \pm 1.1\right)$. The 


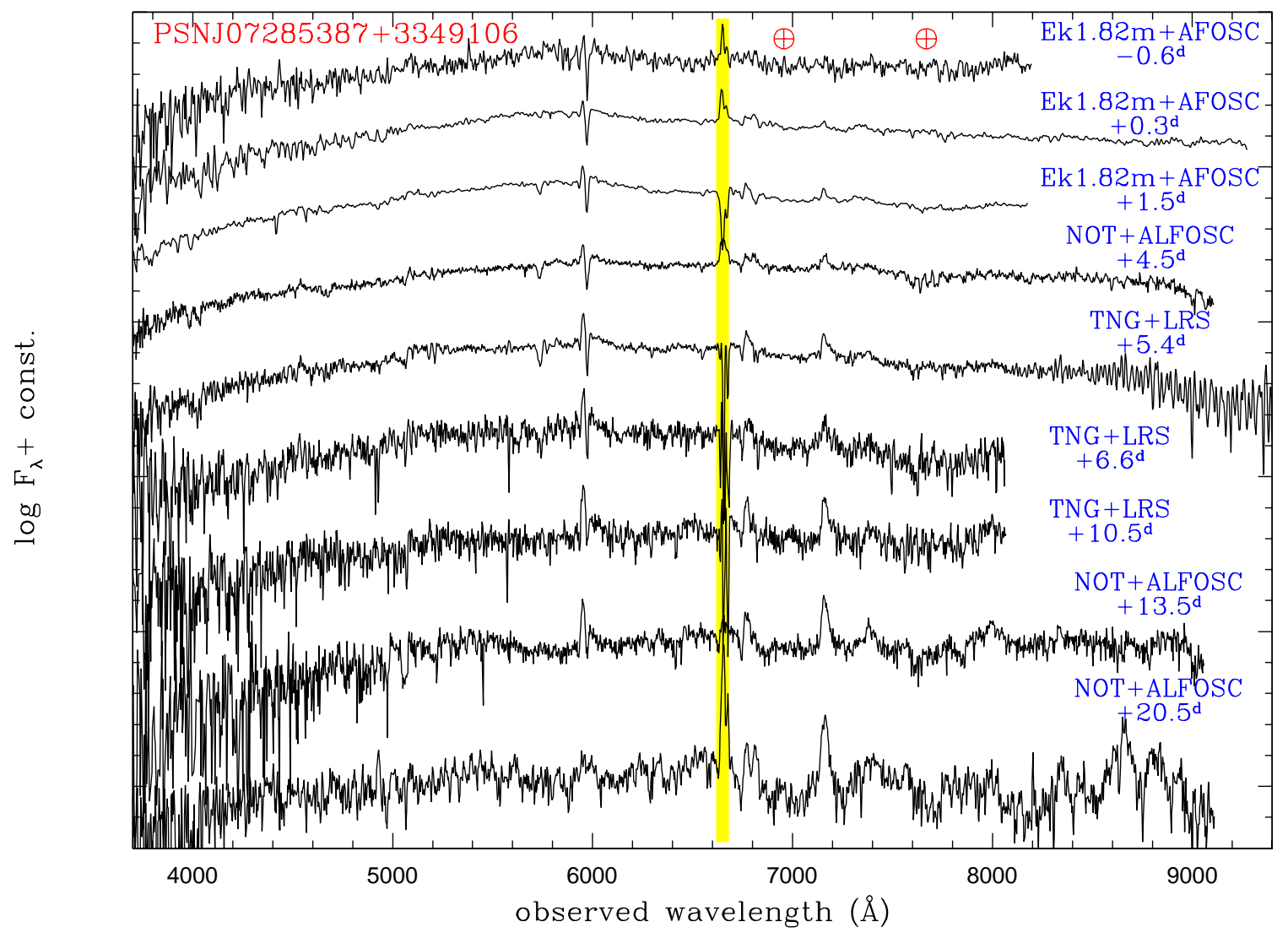

Figure 4. Spectral sequence of PSN J07285387+3349106. The region of $\mathrm{H} \alpha$ is largely contaminated by the narrow $\mathrm{H} \alpha$ and [N II] lines from the host galaxy background (marked by the yellow vertical band). No reddening or redshift corrections have been applied to the spectra.

rise time to maximum in the above two bands amounts to more than $\sim 9 \mathrm{~d}$. After peak, we observe a monotonic decline in all bands, with the light curves in the blue bands fading more rapidly than those in the red bands. The decline rates are moderate in all bands between $0 \mathrm{~d}$ and $+10 \mathrm{~d}$ from maximum: $15.3 \pm 0.3 \mathrm{mag} 100 \mathrm{~d}^{-1}$ in the $u$ band, $9.4 \pm 0.7 \mathrm{mag} 100 \mathrm{~d}^{-1}$ in the $V$ band, and $6.7 \pm$ 1.1 mag $100 \mathrm{~d}^{-1}$ in the $r$ band. From $+10 \mathrm{~d}$ onwards, the declines become much steeper, and are between 28 and $30 \mathrm{mag} 100 \mathrm{~d}^{-1}$ in all bands. The average decline rate of PSN J07285387+3349106 in the $r$ band from the light curve peak to the last detection $(\sim 22 \mathrm{~d}$ after maximum) is $19.7 \pm 1.6 \mathrm{mag} 100 \mathrm{~d}^{-1}$, which is the fastest decline ever observed for a Type Ibn SN in that temporal range.

In Figure 3 (top panel), we show the $B-V$ colour evolution of PSN J07285387+3349106 for two different assumptions for the reddening, i.e. $\mathrm{E}(B-V)_{\text {tot }}=0.99$ mag and $\mathrm{E}(B-V)_{\text {tot }}=0.81$ mag. The colour curves are compared with those of a selected sample of Type Ibn SNe9 including the prototype SN 2006jc and a few objects that share spectroscopic and photometric similarities with PSN J07285387+3349106. While SN 2006jc is blue and shows

\footnotetext{
9 The distance modulus and reddening values adopted for the comparison object are the following: $\mu=36.59$ mag and $\mathrm{E}(\mathrm{B}-\mathrm{V})=0.14 \mathrm{mag}$ for ASASSN-15ed; $\mu=35.27 \mathrm{mag}$ and $\mathrm{E}(\mathrm{B}-\mathrm{V})=0.15 \mathrm{mag}$ for SN $1999 \mathrm{cq} ; \mu$ $=35.52 \mathrm{mag}$ and $\mathrm{E}(\mathrm{B}-\mathrm{V})=0.11 \mathrm{mag}$ for $\mathrm{SN} 2000 \mathrm{er} ; \mu=32.01 \mathrm{mag}$ and $\mathrm{E}(\mathrm{B}-\mathrm{V})=0.04 \mathrm{mag}$ for SN 2006jc; $\mu=34.27 \mathrm{mag}$ and $\mathrm{E}(\mathrm{B}-\mathrm{V})=0.06 \mathrm{mag}$ for SN 2010al.
}

very little $B-V$ colour evolution (see discussion in Pastorello et al. 2015b), other objects of the sample become redder with time up to about four weeks past maximum. Their colour rises from $B-V \approx$ 0 around maximum to about 1 mag four weeks later.

Adopting $\mathrm{E}(B-V)_{t o t}=0.99 \mathrm{mag}$, this would make PSN J07285387+3349106 slightly bluer than similar objects. For this reason, we compute a colour template for Type Ibn SNe through a low-order polynomial fit to the colours of the sample considered in Figure 3 from which we remove SN 2006jc because of its different colour evolution. The best colour match between PSN J07285387+3349106 and the template is obtained with $\mathrm{E}(B-V)_{t o t}=0.81 \pm 0.21$ mag. However, as discussed in Section 3 interacting $\mathrm{SNe}$ such as Type Ibn events may easily have intrinsically different colours, so the colour match among the objects of this sub-sample cannot be used as a conclusive argument to support a lower reddening scenario for PSN J07285387+3349106. For this reason, in Section 3 we conservatively adopted the weighted average of three independent methods as our best reddening estimate, i.e. $\mathrm{E}(B-V)_{t o t}=0.99 \pm 0.48 \mathrm{mag}$.

Accounting for the distance estimate reported in Section 2 and the above reddening value, we obtain the following absolute peak magnitudes in the two bands monitored around the light curve maximum: $M_{V}=-20.30 \pm 1.50$ and $M_{r}=-19.83 \pm$ 1.19. A comparison of the absolute $R$-band magnitudes for the same SN sample as before (with the inclusion of SN 1999cq, Matheson et al. 2000) is shown in the bottom panel of Figure 3 For PSN J07285387+3349106, we adopted the correction of 0.23 

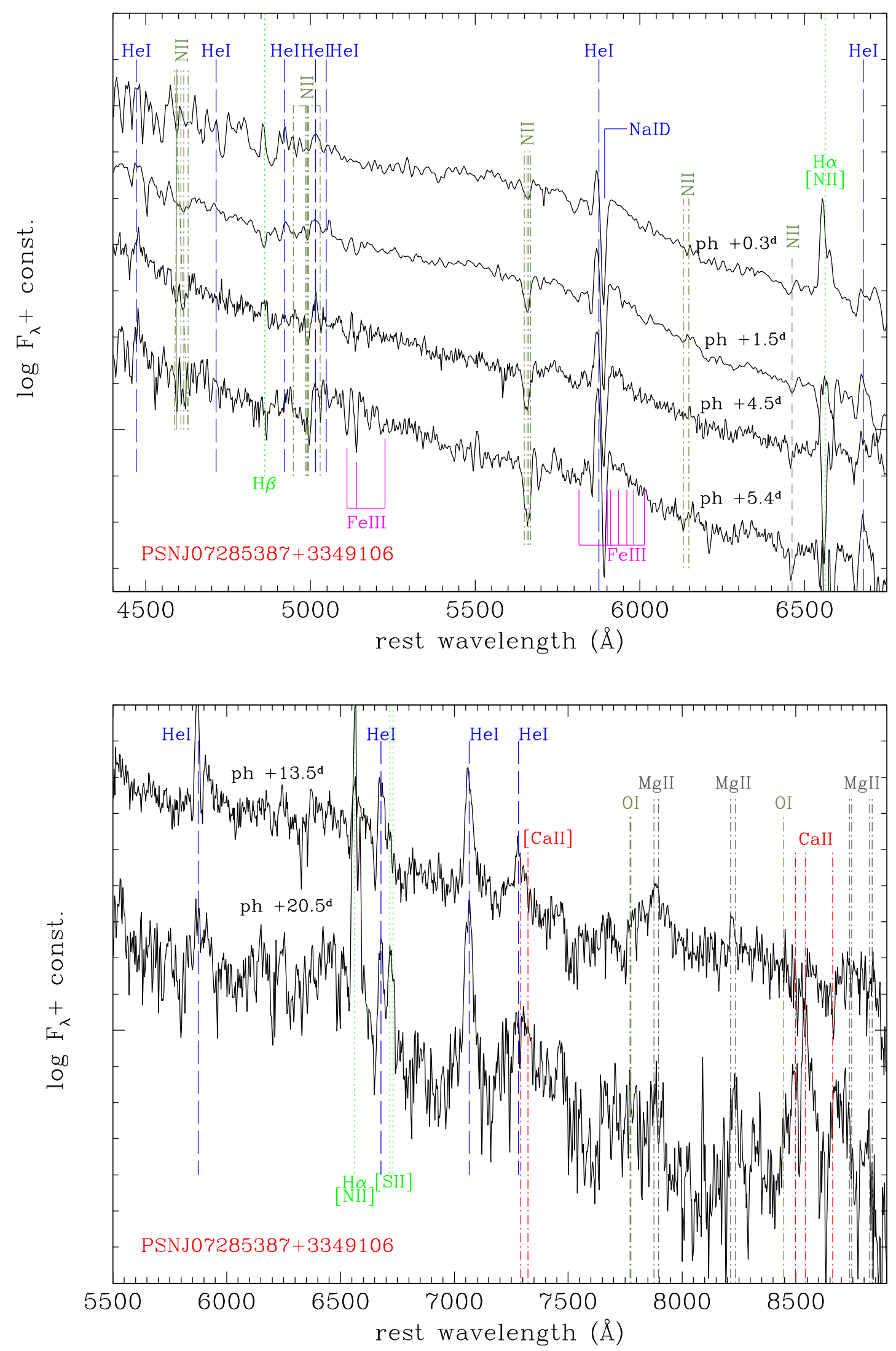

Figure 5. Top - Line identification in early spectra of PSN J07285387+3349106. The Fe III and N II lines are marked at the position of the absorption minimum, blueshifted by $950 \mathrm{~km} \mathrm{~s}^{-1}$ from the rest wavelength position. The He I lines are indicated at rest wavelength. Bottom - Line identification in late spectra of PSN J07285387+3349106. All lines are indicated at the rest wavelength position. The spectra have been corrected for reddening and redshift, using the values adopted in this paper. We also mark the $\mathrm{H} \alpha$ plus [N II] blend, $\mathrm{H} \beta$, and the [S II] $\lambda \lambda 6717,6731$ doublet, which are due to foreground contamination, with green dotted lines. 
mag to convert Sloan r (ABmag) to Johnson-Cousins R (Vegamag) as mentioned in the caption of Figure 2 The SN competes with the most luminous objects in the sample. This comparison shows that there is some similarity in the evolution (in particular, in the decline rates) among the objects that belong to this restricted sample. However, we remark that the general photometric behaviour of SNe Ibn can be strongly heterogeneous. This issue will be discussed in a forthcoming paper (Pastorello et al. 2015e).

\section{SPECTROSCOPIC EVOLUTION}

The sequence of spectra collected for PSN J07285387+3349106 is shown in Figure 4 . We note that there is some residual background contamination in several spectra. A narrow and unresolved $\mathrm{H} \alpha$ emission feature due to unrelated sources in the host galaxy is prominent in spectra obtained with the lowest resolution grisms or under poor seeing conditions. In other cases, a residual absorption is visible at the position of the contaminating $\mathrm{H} \alpha$ line due to an over-subtraction of the background.

The early spectra of PSN J07285387+3349106 show a red, almost featureless continuum, where the most prominent features are due to host galaxy background contamination ( $\mathrm{H} \alpha$, [N II] and [S II] features) which cannot be properly removed. A narrow, unresolved absorption attributed to the $\mathrm{Na}$ ID feature is also clearly detected in our early spectra. This is produced by $\mathrm{Na} \mathrm{I}$ atoms lying along the line of sight inside NGC 2388. However, there is no clear evidence that this absorption is related the SN circumstellar environment. The strength of the interstellar Na ID absorption decreases with time, because a prominent narrow He I $\lambda 5876$ emission feature emerges in its blue wing. Narrow emission lines of He I $\lambda 6678$ (blended, in some early spectra, with the host galaxy [S II] doublet) and $\lambda 7065$ are also detected with P-Cygni profile.

We attempt to identify a number of weak lines visible in the early-phase spectra (see Figure 5, top). In particular, we suggest the unusual detection of Fe III and N II features, with absorption components blueshifted by about $950 \mathrm{~km} \mathrm{~s}^{-1}$. In particular, Fe III $\lambda 5127$ and Fe III $\lambda 5156$ are strong. The identification of Fe III lines at 5800-6000 $\AA$ is ambiguous given the low S/N level. N II blends likely produce some absorption features at about $4620 \AA$, $5650 \AA$ and $6130 \AA$. In addition, we identify N II $\lambda 6482$. Although these lines have not been identified before in early spectra of $\mathrm{SNe}$ Ibn, they have occasionally been detected in massive, hot stars (e.g., Gvaramadze et al. 2010).

In our late spectra, He I $\lambda 7065$ appears to be slightly broader, with a full width at half maximum (FWHM) velocity increasing to about $1300 \mathrm{~km} \mathrm{~s}^{-1}$. In addition, whilst the continuum becomes even redder, other lines appear at the long wavelengths, likely due to O I ( $\lambda 7774-7777$ and $\lambda 8446), \mathrm{Mg}$ II $(\lambda 7877-7896, \lambda 8214$ $8235, \lambda 8735-8745, \lambda 8824-8835)$ and Ca II. In particular, the NIR $\mathrm{Ca}$ II triplet becomes the strongest $\mathrm{SN}$ feature visible in our latest spectrum, while [Ca II] $\lambda \lambda 7291,7324$ is barely detected, blended with He I $\lambda 7281$. Unfortunately, the large reddening, the heavy background contamination and the relatively modest $\mathrm{S} / \mathrm{N}$ of our spectra do not allow to securely identify other spectral lines.

The evolution of the He I $\lambda 5876$ plus Na ID blend (left panel) and the He I $\lambda 7065$ line (right panel) is presented in Figure 6 We note the increasing strength of the He I $\lambda 5876$ line relative to the Na ID foreground absorption, which almost completely disappears in our late spectra. Similarly, the He I $\lambda 7065$ line increases in strength, becoming one of the most prominent $\mathrm{SN}$ features in our late spectra. The velocity of the slow-moving gas, as measured

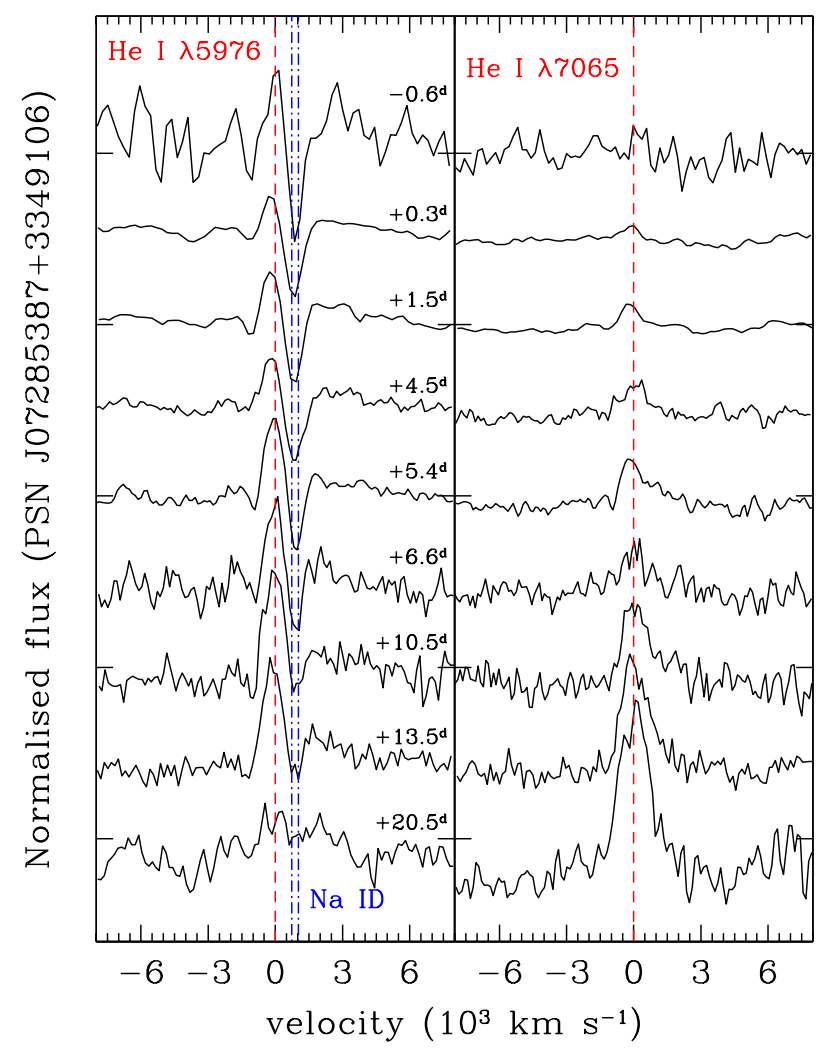

Figure 6. Evolution of the He I $\lambda 5876$ and $\mathrm{Na}$ ID blend (left) and the He I $\lambda 7065$ line (right) in our spectra of PSN J07285387+3349106. The vertical dashed lines mark the rest velocity of the He I lines, while the two dotdashed lines in the left panel mark the position of the two Na ID absorption lines. The velocity scale on the abscissa is in units of $10^{3} \mathrm{~km} \mathrm{~s}^{-1}$.

from the FWHM of the narrow He I $\lambda 7065$ component or from the position of its weak narrow P-Cygni absorption, is about 900-1000 $\mathrm{km} \mathrm{s}^{-1}$. A broader component is also marginally detected in the He I $\lambda 7065$ feature, with an approximate velocity of about 3100 $3400 \mathrm{~km} \mathrm{~s}^{-1}$. This component is best seen in our late time spectra (see e.g. Figure 7).

\section{DISCUSSION}

The analysis of the spectroscopic data of PSN J07285387+3349106 suggests that the very red apparent colour detected at all epochs is a consequence of a relatively large reddening in the direction to the $\mathrm{SN}$. Once corrected for the intrinsic extinction, PSN J07285387+3349106 is a Type Ibn SN with similar properties as already observed in a number of objects of this type.

In particular, the spectrum at early phases is dominated by narrow P-Cygni lines of He I. We propose that these features, with a FWHM velocity of about $900-1000 \mathrm{~km} \mathrm{~s}^{-1}$, are the signature of a circumstellar wind. The velocity of this CSM, along with its chemical composition, may provide important insights into the nature of the progenitor star. This wind is likely $\mathrm{H}$-free, because the unresolved Balmer lines observed in the spectra are attributed to host galaxy contamination.

Specific for this object and unprecedented is the detection of $\mathrm{N}$ II lines in the early spectra. This prompts for a WN-type 


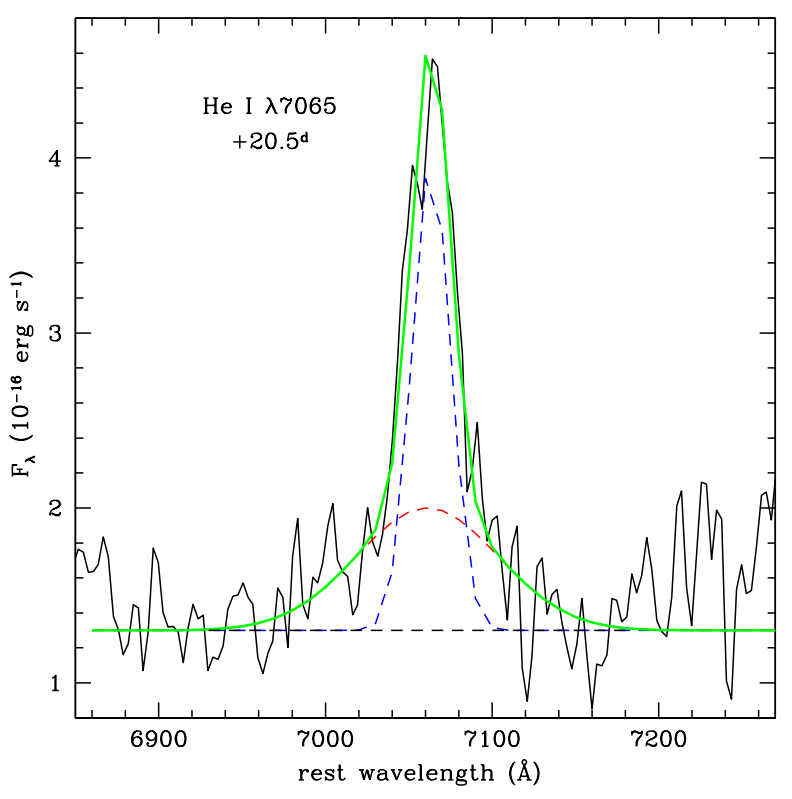

Figure 7. Two-component Gaussian fit to the He I $\lambda 7065$ line in the latest $\mathrm{NOT}+\mathrm{ALFOSC}$ spectrum (phase $+20.5 \mathrm{~d}$ ).

Wolf-Rayet progenitor. More specifically, the lack of circumstellar Balmer lines suggests a WNE subtype classification Hamann et al. 1995). This subclass of Wolf-Rayet stars has a typical terminal wind velocity of about $1600 \mathrm{~km} \mathrm{~s}^{-1}$ (Hamann et al. 2006), consistent with the velocities measured for the bulk of the circumstellar material of PSN J07285387+3349106.

PSN J07285387+3349106 is also a very luminous event (likely brighter than $-20 \mathrm{mag}$, though this estimate is affected by a big uncertainty in the light-of-sight extinction), showing an extremely fast post-peak decline, without a visible flattening onto the ${ }^{56} \mathrm{Co}$ tail. All of this suggests that the luminous peak of the light curve is powered by interaction with CSM rather than a large amount of synthesized ${ }^{56} \mathrm{Ni}$.

A number of authors (e.g., Ofek et al. 2010; Chevalier \& Irwin 2011; Svirski et al. 2012) suggest that a fraction of interacting $\mathrm{SNe}$ have early light curves powered by shock breakout in a dense wind. In fact, while the shock breakout emerging from the stellar surface has a very short duration (of the order of minutes) and releases UV and X-ray photons, the situation changes when the SN explodes within a dense circumstellar cocoon. In that case, the shock may propagate in the CSM without immediately releasing photons to the observer. By the time the forward shock breaks out, it has accumulated a lot of energy which is released over much longer timescales than in the windless case. This mechanism may explain the luminous and long-lasting light curve peaks of a number of interacting $\mathrm{SNe}$ embedded in a dense CSM, as suggested by Ofek et al. (2014a) (but see Moriya \& Maeda 2014). The shock breakout model in a dense circumstellar wind has the advantage of explaining the enormous peak luminosity of PSN J07285387+3349106, along with its relatively rapid photometric evolution with respect to the very slow evolutionary time scales of more extreme interacting $\mathrm{SNe}$ (e.g. Aretxaga et al. 1999).

A potential problem of this interpretation is the requirement of high CSM densities. In order to produce such a dense CSM, the precursor star should have high mass-loss rate, from a few $10^{-3}$
$\mathrm{M}_{\odot} \mathrm{yr}^{-1}$ to $10^{-1} \mathrm{M}_{\odot} \mathrm{yr}^{-1}$ (Kiewe et al. 2012; ; Taddia et al. 2013; Moriva et al. 2014). These substantial mass-loss rates are expected in luminous blue variable (LBV) eruptions, and not in steady winds of the putative Wolf-Rayet progenitors of SNe Ibn (Hamann et al. 2000; Nugis \& Lamers 2000). Nonetheless, at least in the case of SN 2006jc, a residual LBV-like behaviour has been observed through the detection of a pre-SN burst in 2004 (Nakano et al. 2006; Pastorello et al. 2007). A rather complex CSM density profile and a variable mass-loss rate, ranging from $10^{-1} \mathrm{M}_{\odot} \mathrm{yr}^{-1}$ during the outburst to many orders of magnitude less before and after the 2004 episode, were proposed by Tominaga et al. (2008). We emphasise that the shock breakout scenario in an optically thick CSM has also been suggested for the Type Ibn SN iPTF13beo (Gorbikov et al. 2014). The shock breakout interpretation for this $\mathrm{SN}$ requires an enormous mass-loss (about $2.4 \mathrm{M}_{\odot} \mathrm{yr}^{-1}$ ) over a very short period. In other words, though unobserved, an LBVlike violent eruption is necessary also to explain the properties of iPTF13beo. The ultimate verification of this scenario is the detection of pre-SN eruptions. So far, SN 2006jc is the only SN Ibn for which a pre-SN eruption has been registered. However, pre-SN observations of the host galaxies are not available for most Type Ibn $\mathrm{SNe}$, or these $\mathrm{SNe}$ are too distant to detect $\sim-14$ mag pre-SN transient events.

It is evident that routine observations of so-called "SN impostors" (Van Dyk et al. 2000; Maund et al. 2006; Pastorello et al. 2010, 2013; Tartaglia et al. 2015a; Kankare et al. 2015) are essential to monitor extremely rare very massive stars that are approaching their final death as luminous SNe. This strategy is complemented by panoramic surveys such as the intermediate Palomar Transient Factory, La Silla-QUEST, Pan-STARRS, the Catalina Real-Time Transient Survey, and the SkyMapper Southern Sky Survey which provide extensive databases of future SN explosion sites. These images will allow us to recover a posteriori the variability history of the progenitors of new interacting $\mathrm{SNe}$, as done for some recent SNe IIn (Fraser et al. 2013; Ofek et al. 2014b; Tartaglia et al. 2015b).

\section{ACKNOWLEDGEMENTS}

We are grateful to Massimo Conti, Giacomo Guerrini, Paolo Rosi, Luz Marina Tinjaca Ramirez for their help with the observations. We are also grateful to Manfred Mrotzek (http://www.astro-photos.net/index_en.htm) and Paolo Campaner (http://paolocampaner.blogspot.it) for providing their observations of PSN J07285387+3349106. Part of the observations in Asiago were done during the Opticon/Neon school (www.iap.fr/neon/), by the PhD students Demetra de Cicco (Napoli), Marco Lam (Edimburgh), Ignacio Ordovas (Santander) and Paulina Sowicka (Warsaw), under the supervision of Nancy Elias-Rosa, and Michel Dennefeld. Opticon is funded by the European Commission's FP7 Capacities programme (Grant number 312430).

AP, SB, NER, AH, LT, GT, and MT are partially supported by the PRIN-INAF 2014 with the project "Transient Universe: unveiling new types of stellar explosions with PESSTO". NER acknowledges the support from the European Union Seventh Framework Programme (FP7/2007-2013) under grant agreement n. 267251 "Astronomy Fellowships in Italy" (AstroFIt). AMG acknowledges financial support by the Spanish Ministerio de Economía y Competitividad (MINECO) grant ESP2013-41268-R. ST and UMN acknowledge support by TRR33 "The Dark Universe" of the German Research Foundation (DFG). EEOI is partially supported by 
the Brazilian agency CAPES (grant number 9229-13-2). We gratefully acknowledge the support from the Knut and Alice Wallenberg Foundation. The Oskar Klein Centre is funded by the Swedish Research Council.

This paper is based on observations made with the Italian Telescopio Nazionale Galileo (TNG) operated on the island of La Palma by the Fundación Galileo Galilei of the INAF (Istituto Nazionale di Astrofisica) and the Copernico Telescope of INAF-Asiago Observatory and the Rapid Eye Mount (REM) INAF telescope hosted at La-Silla (European Southern Observatory, Chile). This work is also based on observations obtained with the Nordic Optical Telescope, which is operated by the Nordic Optical Telescope Scientific Association at the Observatorio del Roque de los Muchachos, La Palma, Spain, of the Instituto de Astrofisica de Canarias. This paper is also based on observations obtained with the Joan Oró Telescope (TJO) of the Montsec Astronomical Observatory (OAdM) which is owned by the Catalan Government and operated by the Institute for Space Studies of Catalonia (IEEC).

This research has made use of the NASA/IPAC Extragalactic Database (NED) which is operated by the Jet Propulsion Laboratory, California Institute of Technology, under contract with the NASA. We acknowledge the usage of the HyperLeda database (http://leda.univ-lyon1.fr).

\section{REFERENCES}

Anupama, G. C., Sahu, D. K., Gurugubelli, U. K., Prabhu, T. P., Tominaga, N., Tanaka, M., Nomoto, K., 2009, MNRAS, 392, 894

Aretxaga, I., Benetti, S., Terlevich, R. J., Fabian, A. C., Cappellaro, E., Turatto, M., della Valle, M., 1999, MNRAS, 309, 343

Bianco, F. B. et al., 2014, ApJS, 213, 19

Brown, M. J. et al., 2014, ApJS, 212, 18

Calzetti, D., Kinney, A. L., Storchi-Bergmann, T., 1994, ApJ, 429, 582

Cappellaro, E., 2014, "SNOoPy: a package for SN photometry", http://sngroup.oapd.inaf.it/snoopy.html

Cardelli, J. A., Clayton, G. C., Mathis, J. S., 1989, ApJ, 345, 245

Chevalier, R. A., Irwin, C. M., 2011, ApJ, 729, L6

Chonis, T. S., Gaskell, C. M., 2008, AJ, 135, 264

Chugai, N. N. et al. 2004, MNRAS, 532, 1213

Chugai, N. N., 2009, MNRAS, 400, 866

Di Carlo, E. et al., 2008, ApJ, 684, 471

Foley, R. J., Smith, N., Ganeshalingam, M., Li, W., Chornock, R., Filippenko, A. V., 2007, ApJ, 657, L105

Fraser, M. et al. 2013, ApJ, 779, L8

Fraser, M. et al. 2015, MNRAS, to be submitted

Gorbikov, E. et al., 2014, MNRAS, 443, 671

Gvaramadze, V. V. et al., 2010, MNRAS, 405, 520

Hamann, W.-R., Koesterke, L., Wessolowski, U., 1995, A\&A, 299, 151

Hamann, W.-R., Koesterke, L., Gräfener, G., 2000, in Thermal and Ionization aspects of Flows from Hot Stars: Observations and Theory, ed. H. J. G. L. M. Lamers, \& A. Sapar, ASP Conf. Ser., 204, 197

Hamann, W.-R. , Gräfener, G., Liermann, A., 2006, A\&A, 457, 1015

Immler, S. et al., 2008, ApJ, 674, L85

Kankare, E. et al. 2012, MNRAS, 424, 855

Kankare, E. et al. 2015, A\&A, submitted

Kiewe, M. et al., 2012, ApJ, 744, 10
Makarov, D., Prugniel, P., Terekhova, N. Courtois, H. Vauglin, I., 2014, A\&A, 570, 13

Matheson, T., Filippenko, A. V., Chornock, R., Leonard, D. C., Li, W., 2000, AJ, 119, 2303

Mattila, S. et al., 2008, MNRAS, 389, 141

Maund, J. R. et al., 2006, MNRAS, 369, 390

Modjaz, M. et al., 2014, AJ, 147, 99

Moriya, T. J., Maeda, K., Taddia, F., Sollerman, J., Blinnikov, S.I, Sorokina, E. I., 2014, MNRAS, 439, 2917

Moriya, T. J., Maeda, K., 2014, ApJ, 790, L16

Nakano, S., Itagaki, K., Puckett, T., Gorelli, R., 2006, CBET, 666, 1

Nozawa, T. et al., 2008, ApJ, 684, 1343

Nugis, T., Lamers, H. J. G. L., M. 2000, A\&A, 360, 227

Ochner, P. et al., 2015, Astron. Tel. 7105

Ofek, E. et al. 2010, ApJ, 724, 1396

Ofek, E. et al. 2014a, ApJ, 788, 154

Ofek, E. et al. 2014b, ApJ, 789, 104

Pastorello, A., et al. 2007, Nature, 447, 829

Pastorello, A. et al., 2008a, MNRAS, 389, 113

Pastorello, A. et al., 2008b, MNRAS, 389, 131

Pastorello, A. et al., 2013, MNRAS, 408, 181

Pastorello, A. et al., 2013, ApJ, 767, 1

Pastorello, A. et al., 2015a, MNRAS, 449, 1921

Pastorello, A. et al., 2015b, MNRAS, 453, 3649

Pastorello, A. et al., 2015c, MNRAS, 449, 1954

Pastorello, A. et al., 2015d, MNRAS, 449, 1941

Pastorello, A. et al., 2015e, MNRAS, submitted (eprint arXiv: submit/1365375)

Phillips, M. M., Lira, P., Suntzeff, N. B., Schommer, R. A., Hamuy, M., Maza, J., 1999, AJ, 118, 1766

Pilyugin, L. S., Vílchez, J. M., Contini, T., 2004, A\&A, 425, 849

Poznanski, D., Ganeshalingam, M., Silverman, J. M., Filippenko, A. V., 2011, MNRAS, 415, L81

Sakon, I. et al. 2009, ApJ, 692, 546

Sanders, D. B., Mazzarella, J. M., Kim, D.-C., Surace, J. A., Soifer, B. T., 2003, AJ, 126, 1607

Sanders, N. E. et al., 2013, ApJ, 769, 39

Schlafly, E. F., Finkbeiner, D. P., 2011, ApJ, 737, 103

Smith, N., Foley, R. J., Filippenko, A. V., 2008, ApJ, 680, 568

Smith, N., Mauerhan, J. C., Silverman, J. M., Ganeshalingam, M., Filippenko, A. V., Cenko, S. B., Clubb, K. I., Kandrashoff, M. T., 2012, MNRAS, 426, 1905

Stritzinger, M. et al. 2012, ApJ, 756, 173

Svirski, G., Nakar, E., Sari, R., 2012, ApJ, 759, 108

Taddia, F. et al., 2013, A\&A, 555, 10

Taddia, F. et al., 2015, A\&A accepted (arXiv:1505.04719)

Tartaglia, L. et al. 2015a, MNRAS, 447, 117

Tartaglia, L. et al. 2015b, MNRAS, submitted

Tominaga, N. et al., 2008, 687, 1208

Tremonti, C. A. et al., 2004, ApJ, 613, 898

Turatto, M., Benetti, S., Cappellaro, E., 2003, From Twilight to Highlight: The Physics of Supernovae: Proceedings of the ESO/MPA/MPE Workshop Held at Garching, Germany, 29-31 July 2002, ESO ASTROPHYSICS SYMPOSIA. Edited by W. Hillebrandt and B. Leibundgut. Springer-Verlag, p. 200

Tsvetkov, D. Yu., Volkov, I. M., Pavlyuk, N. N., 2015, Information Bulletin on Variable Stars, 6140, 1

Van Dyk, S. D., Peng, C. Y., King, J. Y., Filippenko, A. V., Treffers, R. R., Li, W., Richmond, M. W., 2000, PASP, 112, 1532

White, R. A., Bliton, M., Bhavsar, S. P., Bornmann, P., Burns, J. O., Ledlow, M. J., Loken, C., 1999, AJ, 118, 2014 
Wright E. L., 2006, PASP, 118, 1711

Zwicky, F., Herzog, E., Karpowicz, M., Kowal, C. T., Wild, P., 1961-1968, Catalogue of Galaxies and of Clusters of Galaxies (6 vols. ; Pasadena: Caltech)

This paper has been typeset from a $\mathrm{T}_{\mathrm{E}} \mathrm{X} / \mathrm{LT}_{\mathrm{E}} \mathrm{X}$ file prepared by the author. 